\title{
BII. MODELING OF THE CD20 AND CD52 MEMBRANE RECEPTORS AND STUDY OF THEIR INTERACTION WITH DMPC MEMBRANE BY MOLECULAR DYNAMIC SIMULATION.
}

Marcos Roberto Lourenzoni ${ }^{1}$; Alison de Sousa Rebouças ${ }^{1}$; Natália Fernandes Frota $^{1}$; Ana Virgínia Frota Guimarães ${ }^{1}$; Gabriel Nascimento Maranhão ${ }^{1}$; Maurício Cardoso da Costa Júnior ${ }^{1}$; Claudia do Ó Pessoa ${ }^{1}$.

${ }^{1}$ Fundação Oswaldo Cruz - CE.

INTRODUCTION Cancer is one of the main causes of mortality in the world according to the World Health Organization. Thus, studies of alternatives for cancer treatment are extremely important, such as, the use of more effective monoclonal antibodies (mAbs). CD20 and CD52 are membrane receptors that are overexpressed in leukemia and lymphoma cells, which makes them ideal targets for $\mathrm{mAbs}$, for example, the commercial antibodies Rituximab and Campath- $1 \mathrm{H}$. There is no crystallographic structure available for these receptors, which limits a better understanding of their interaction with $\mathrm{mAbs}$ and the biological membrane.

OBJECTIVE To obtain three-dimensional structure of CD20 and CD52 receptors in a model membrane represented by a dimyristoyl phosphatidylcholine (DMPC) bilayer.

METHODOLOGY Four CD20 transmembrane helices were modeled in the Swiss-PdbViewer software, and then submitted to molecular dynamics (MD) simulation immersed in $\mathrm{n}$-hexane/water interface. CD20 was modeled by inserting the polypeptide chains that connect the helices. Then, it was inserted into the DMPC bilayer followed by submission to DM simulation for $100 \mathrm{~ns}$. CD52 structure contains a 12 amino acid peptide and a GPI anchor, which was drawn in the Accelrys Draw 4.2 software and then submitted to the Automated Topology Builder (ATB) and Repository program for obtaining a three-dimensional structure and a force field compatible with GROMOS53a6 force field.The CD52 was obtained by unifying the GPI and the peptide topologies for the same force field, and submitted to DM simulation immersed in DMPC bilayer, interfaced by water. The CD20 and CD52 structural stability was evaluated by root mean square deviation (RMSD) in Gromacs 5.0.2 software. 
RESULTS CD20-loop structure that binds to Rituximab stabilizes after 27 ns of simulation with RMSD of $\sim 0.09 \mathrm{~nm}$. The structure of the CD20 epitope reaches equilibrium after $25 \mathrm{~ns}$ (RMSD of $\sim 0.02 \mathrm{~nm}$ ). In the CD52 structure, the protein portion stabilizes after $10 \mathrm{~ns}(\mathrm{RMSD} \sim 0.40 \mathrm{~nm})$ and the region that defines the epitope admits RMSD of $\sim 0.15 \mathrm{~nm}$.

CONCLUSION The CD20 and CD52 models show a three dimensional structural organization in greater detail than the epitope only, which is normally used for obtaining the antibody. The small fluctuations in RMSD, after CD20 and CD52 reach equilibrium in $\mathrm{MD}$, support the epitope function of being the most complementary region with mAbs, defining the antibody-antigen specificity. These models are validated for the continuity of the project of Rituximab and Campath$1 \mathrm{H}$ biobetters development, considering more realistic models of CD20 and CD52. Acknowledgments: Fiocruz, Funcap, CNPq, Centro Nacional de Processamento de Alto Desempenho (Cenapad) - Ceará.

KEYWORDS CD20, CD52, cancer, molecular dynamics. 